\title{
Shunt Analysis in the Isolated-Target Divertor Model for Plasma Detachment Measurement in DEMO
}

\author{
Samir El Shawish \\ Jožef Stefan Institute, Jamova 39, SI-1000, Ljubljana, Slovenia \\ Louis Giannone \\ Max-Planck-Institut für Plasmaphysik, Boltzmannstrasse 2, D-85748 Garching, Germany
}

\begin{abstract}
A recently proposed isolated-target design solution for the DEMO divertor introduces a shunt resistor between the target plate and the cassette body, which are otherwise electrically isolated from each other. Such a design serves as a diagnostic tool for measuring thermo-currents (voltages) through a shunt to control plasma detachment. Ideally, large shunt resistances would be desired in order to provide measurable voltage signals. However, during global plasma instabilities, very large electrical currents may be induced between the target plates which need to be routed through a shunt towards the massive cassette body to avoid damage of the cooling pipes. An optimal shunt resistance is therefore required to provide measurable thermo voltage signals and to protect the piping from the effects of most extreme plasma instabilities.

In this study, a steady-state finite-element analysis is performed for the latest divertor geometry design assuming realistic extreme loading conditions of VDE-IV slow down with 30/89 $\mathrm{kA}$ applied on the inner/outer plasma facing components and channel-like pipe fixations that prevent pipe bending. In particular, the number of shunts, their maximum resistances and optimal locations are proposed from the requirement that the temperature of the water-cooling pipes does not exceed the water boiling temperature and that all the pipes remain their structural integrity during the VDE-IV disruption. The results of the analysis suggest that four shunts of $\sim 600 \mu \Omega$ will protect the pipes during the VDE-IV event and provide a $\sim 64 \mathrm{mV}$ signal during the assumed 150/150 A thermo-current measurement.
\end{abstract}


Keywords:

thermo current, shunt resistance, electromagnetic forces, divertor, DEMO

\section{INTRODUCTION}

The heat load on the plasma facing components (PFC) of target plates in DEMO divertor must be controlled to avoid damage. In the existing

tokamaks this is achieved by measuring the thermo-current flowing to the PFC target plates and using the impurity gas puffing to reduce the heat load to the targets $[1,2]$.

Thermo-current is driven by a temperature difference between the inboard and outboard PFC target plates which causes a current flow to compensate the difference in the sheath potentials $[3,4,5]$. In DEMO this current is estimated to have a steady state value of approximately $150 \mathrm{~A}$ per divertor when plasma attachment begins [6]. When the plasma is fully detached, however, no current should run on average through the divertor.

One of the currently considered design options for thermo-current measurement in DEMO is an isolated-target design solution [7] which involves mounting of an isolated PFC target plate so that the applied current flows partially through a tube of resistive material (a shunt) towards the cassette body and partially through the water-cooling pipes. To maximize the signal to noise ratio for the measured shunt voltage, the shunt resistance should be as large as possible, assuming electrical isolation of the water-cooling pipes from the divertor cassette to feed the thermo-current through a single resistive element. However, due to safety reasons, the shunt resistance should be limited in practice. This limitation turns out to be necessary in the event of plasma disruption to protect the cooling pipes from high currents and, consequently, from high induced mechanical stresses that can emerge in an external magnetic field and consequently reduce the structural integrity of the pipes.

In the case of vertical displacement events (VDEs), the induced halo currents through the divertor can be significantly larger than the thermocurrent itself. The most conservative estimation follows from the nominal plasma current of 19.6 MA redistributed evenly over 48 divertor cassette modulus. In this extreme limit, the maximum VDE current of $410 \mathrm{kA}$ per divertor can in principle be applied. However, this extreme theoretical value has been recently refined to a much lower level. The refined extreme loading 
condition for the divertor is labeled VDE-IV slow down and provides $30 \mathrm{kA}$ and $89 \mathrm{kA}$ halo currents flowing on inner and outer PFC targets, respectively [8].

One of the few feasibility studies $[6,9]$ on thermo-current measurement in isolated-target divertor model in DEMO was presented in our previous work [6]. In that study a finite-element (FE) electro-mechanical analysis was conducted to calculate the conservative upper value of shunt resistance. That value, estimated to $\sim 40 \mu \Omega$, was derived from the requirement that, in case of the most extreme VDE plasma disruption event (with $410 \mathrm{kA}$ applied per divertor), the fraction of the electrical current flowing through the watercooling pipes would result in the loss of their structural integrity. The later was conservatively attributed to the onset of plasticity, which was developed due to excessive pipe bending under the assumption of simplified point-like pipe fixation. The obtained shunt resistance of $\sim 40 \mu \Omega$ was also estimated to provide a thermo-current signal of $\sim 5 \mathrm{mV}$, which seems to be on the lower limit for reliable plasma detachment detection.

The objective of this study is to reduce the conservatism used in our first analysis [6] in order to enhance the thermo-current shunt signal while still retaining the integrity of the water-cooling pipes. This is achieved through several mechanical, thermal and electrical FE analyses by (i) using a recently upgraded isolated-target divertor model $[10,11]$ with optimized pipe fixation system, where water-cooling pipes are firmly embedded (and thus not allowed to bend) in specially designed cassette channels (see Fig. 1), and (ii) considering the refined extreme loading conditions of VDE-IV slow down disruption with $30 \mathrm{kA}$ and $89 \mathrm{kA}$ halo currents flowing on the inner and outer PFC targets, respectively [8]. Providing that water-cooling pipes retain their structural integrity and do not overheat above the water boiling temperature during the VDE-IV event, a much higher shunt voltage of $\sim 64 \mathrm{mV}$ is finally predicted for the $150 \mathrm{~A}$ thermo-current flowing on the target plates.

The paper is organized as follows. Section 2 introduces the finite-element model with all relevant assumptions employed in the electrical analysis. Section 3 provides admissible electrical current densities for different pipe segments using mechanical (Sec. 3.1) and thermal (Sec. 3.2) considerations. Section 4 presents the results of the electrical analysis with identified maximum shunt resistance and thermo-current voltage signal. Finally, the conclusions are given in Sec. 5 . 


\section{FINITE-ELEMENT MODEL FOR ELECTRICAL ANALYSIS}

A 3D steady-state electrical analysis is carried out following Ohm's law to obtain electrical current distributions $j(r)$ inside the divertor cassette body and cooling pipes for the assumed surface current loads. The FE modelling and simulations are performed with Abaqus [12] using an updated isolated-target divertor model DEMO baseline 2017. Contrary to the previous modelling strategy [6], the cassette body and water-cooling pipes are modelled jointly within the same FE model. Electrical conduction between the two parts is modelled at several (4) locations by introducing electrical surface-surface interaction to model shunt elements with adjustable resistance (Fig. 1).

\subsection{Assumptions}

Analysis approximations:

- Thermal effects such as Joule heating are neglected in the calculation of current distributions. Electrical material properties are considered at constant temperature of $200^{\circ} \mathrm{C}$. Joule heating is, however, considered separately in the thermal analysis of water-boiling conditions (see Sec. 3.2).

Geometry:

- The geometry of the isolated-target divertor design follows DEMO baseline 2017 geometry with lateral divertor cassette design [10, 11], see Fig. 1. This design assumes the embedment of the water-cooling pipes into special cassette channels, which practically prevent the bending of the pipes due to induced Lorentz forces.

- The PFC tungsten monoblocks are omitted from the 3D model. However, their influence is considered in a separate structural analysis of damage initiation in PFC pipes (see Sec. 3.1).

Materials (see also Tab. 1):

- The cassette body material is assumed to be Eurofer97 with isotropic electrical resistivity of $0.70 \mu \Omega \mathrm{m}$ at $200^{\circ} \mathrm{C}$ [13]. 


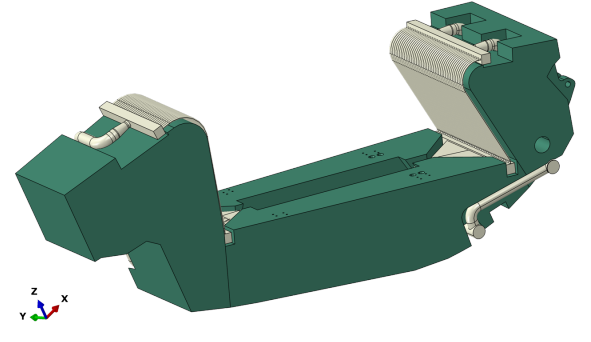

(a)

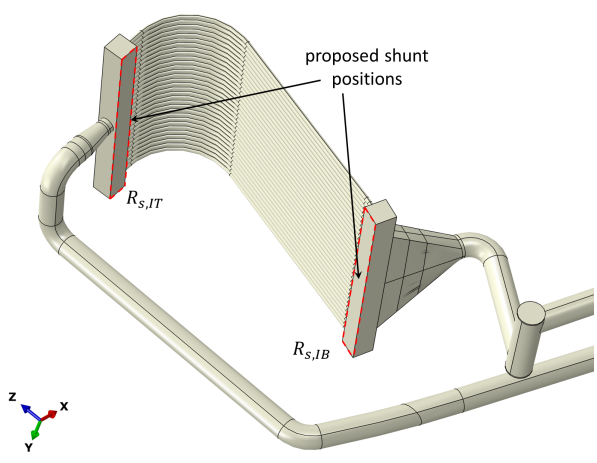

(c)

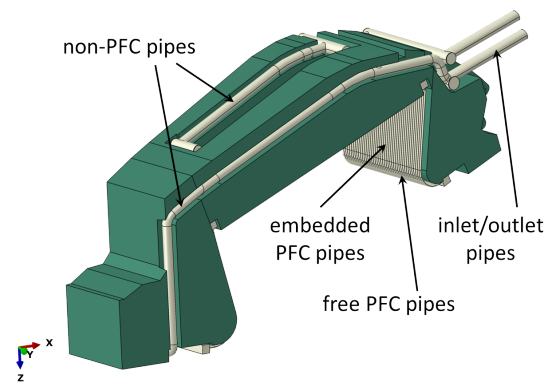

(b)

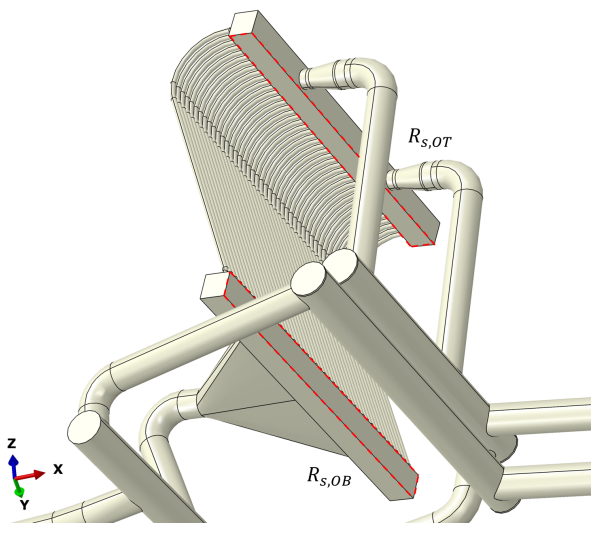

(d)

Figure 1: DEMO baseline 2017 isolated-target divertor model with lateral divertor cassette design. PFC monoblocks are omitted from the model. A shunt element is modelled implicitly as an interaction between the two surfaces located on a cassette body (green) and water-cooling pipes (grey). Four such surfaces are sketched on the pipe model (red).

- The water-cooling pipes are assumed to be composed of two different materials [7]: $\mathrm{CuCrZr}$ in the PFC region and stainless steel AISI-316L in the non-PFC region, with isotropic electrical resistivity of $0.04 \mu \Omega \mathrm{m}$ at $200^{\circ} \mathrm{C}[14]$ and $0.90 \mu \Omega \mathrm{m}$ at $200^{\circ} \mathrm{C}$ [15], respectively ${ }^{1}$.

- The water inside the pipes is assumed to be a perfect electric insulator. This is a conservative but reasonable assumption based on the fact that water resistivity ranges from $\sim 20 \Omega \mathrm{m}$ (drinking water) to $\sim 180 \mathrm{k} \Omega \mathrm{m}$

\footnotetext{
${ }^{1}$ Since temperature dependent electrical resistivity data for AISI-316L stainless steel was unavailable in the literature, the corresponding AISI-316 data was used instead.
} 


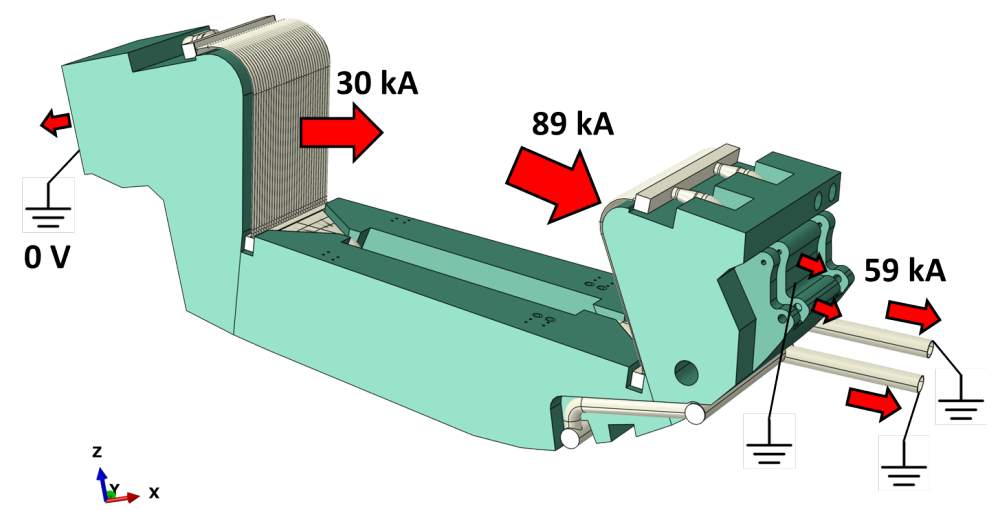

Figure 2: Schematic model of the VDE-IV slow down loading conditions with indicated zero potential regions representing the electrical grounding at both pipe ends and cassette body attachments.

Table 1: Electrical resistivity data at $200^{\circ} \mathrm{C}$ for the materials used in the electrical analysis taken from Refs. [13, 14, 15].

\begin{tabular}{ll}
\hline Material & Resistivity $(\mu \Omega \mathrm{m})$ \\
\hline Eurofer97 & 0.70 \\
AISI-316 & 0.90 \\
CuCrZr & 0.04 \\
\hline
\end{tabular}

(ultrapure water) at $25^{\circ} \mathrm{C}[16]$. However, even if sea water, with resistivity of $\sim 0.2 \Omega \mathrm{m}$ at $25^{\circ} \mathrm{C}[16]$, is considered as an extreme example (to account for, e.g., neutron irradiation effects), this still amounts to at least $10^{5}$ times higher resistivity compared to AISI-316L pipe.

Boundary conditions:

- Zero potential is assigned to both inner and outer attachment regions of the cassette model to represent ideal electrical connection with the vacuum vessel which is assumed to be electrically grounded. Zero potential is also applied to both far-ends of the water-cooling pipes representing water inlet and outlet (Fig. 2).

Loading conditions:

- As tungsten monoblocks are not considered in the 3D FE model, the 
loading currents are applied directly to PFC pipe surfaces (half of the pipe surfaces pointing towards the plasma).

- To account for plasma attachment scenario, a thermo-current of $150 \mathrm{~A}$ is applied uniformly over the inner and outer target plate regions of the cooling pipes [6].

- To account for maximum possible electrical currents, a VDE-IV slow down case [8] is used with $30 \mathrm{kA}$ and $89 \mathrm{kA}$ halo currents flowing through the inner and outer target plates, respectively. Both values are applied uniformly over the inner and outer target plate regions of the cooling pipes (Fig. 2). Due to asymmetry in the loading, the remaining $59 \mathrm{kA}$ current must flow to the ground (set as zero potential), through the pipe ends (inlet and outlet) and through cassette body attachments.

Shunt model:

- The shunt element is modelled with a surface-surface interaction between a surface defined on a cassette body part and a surface defined on a water-cooling pipe part (marked as a red rectangle in Fig. 1). For a given electrical conductance $\sigma_{s}$ between the two surfaces, the effective shunt resistance is estimated as $R_{s}=1 / A \sigma_{s}$ where $A$ is the area of the smaller surface in the surface-surface pair. In all four shunt cases, the smaller surfaces are always located on the pipe part (rectangular PFC joining region) with $A_{\text {in }} \approx 5410^{3} \mathrm{~mm}^{2}$ and $A_{\text {out }} \approx 7510^{3} \mathrm{~mm}^{2}$ on inner and outer sides, respectively. Different $R_{s}$ values are considered in the FE simulations, ranging from $1 \mu \Omega$ to infinity (perfect isolation).

Finite element mesh:

- FE mesh (Fig. 3) is composed of 5557k elements in the cassette body part (linear tetrahedral elements of type DC3D4E) and 543k elements in the water-cooling pipes part (157k linear hexahedral elements of type DC3D8E and 386k linear tetrahedral elements of type DC3D4E). The FE mesh has been estimated to provide reasonably accurate results.

\section{ADMISSIBLE PIPE CURRENTS}

Before proceeding to the 3D electrical analysis, several mechanical and thermal analyses are performed first in order to identify the admissible electrical currents flowing through different regions of the water-cooling pipes. 


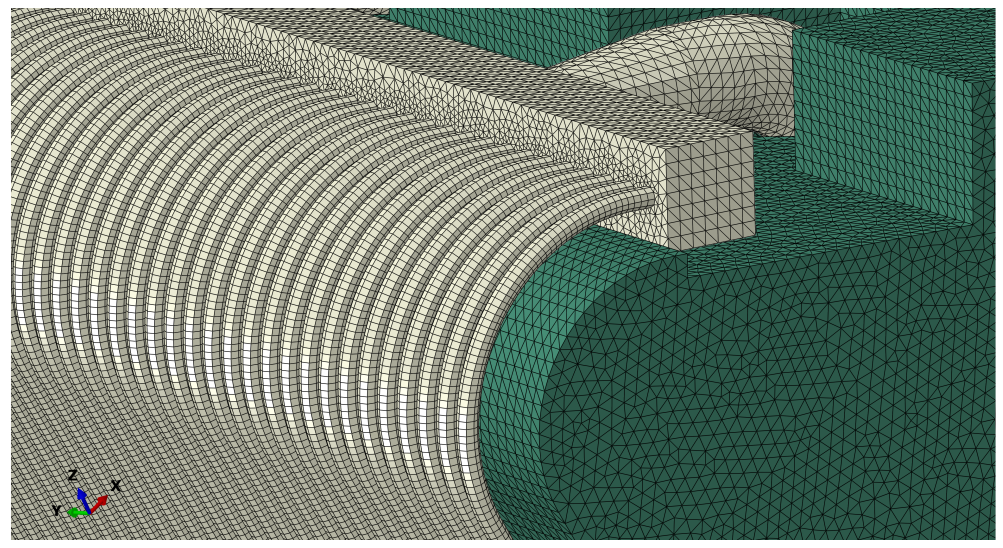

Figure 3: Mesh of the 3D FE model employed for the electrical analysis.

These critical currents will later allow us to identify optimal shunt resistances which will provide measurable thermo voltage signals and protect the piping from the effects of the most extreme plasma instabilities.

\subsection{Mechanical considerations}

In the present geometry, it is assumed that water-cooling pipes are firmly embedded in the cassette body (Fig. 1), which prevents bending of long pipe segments even at extreme loading conditions ${ }^{2}$. For this reason, a special emphasis is put here to identifying other feasible mechanical constraints that will affect the integrity of the pipes. Besides (local) bending of the monoblockfree PFC pipes in the upper target region, the main concern addressed here is a uniform pipe compression against the cassette body (or monoblock in the PFC target region) due to induced magnetic forces within the pipe wall.

To estimate critical magnetic force densities for damage initiation in different pipe locations, four separate FE mechanical simulations are conducted on four different pipe segments representative of the water-cooling pipe model, see Fig. 1(b). In this respect, pipe compression is investigated in two simply-supported pipes (labeled non-PFC pipe and inlet/outlet pipe ${ }^{3}$ ) and in a PFC pipe embedded in tungsten monoblock (labeled embedded PFC pipe). Local pipe bending is analysed only in a monoblock-free segment of

\footnotetext{
${ }^{2}$ Note that pipe bending was identified to be the limiting factor in the previous divertor design where a point-like pipe fixation was assumed within the studied model [6].

${ }^{3}$ Note that non-PFC pipe and inlet/outlet pipe differ only by pipe radius.
} 
the PFC pipe (labeled free PFC pipe). Each pipe model is discussed separately below using the material properties of the pipes and their relevant surrounding structures listed in Tab. 2.

Table 2: Elastic-ideal-plastic material properties [17] at different temperatures $T$ used in the mechanical simulations: elastic modulus $E$, Poisson number $\nu$, (minimum) yield stress $\sigma_{y}$. In the embedded PFC pipe model, one temperature is conservatively assumed for one material, based on the calculated temperature field shown in Fig. 3 of Ref. [18].

\begin{tabular}{llllll}
\hline Region & Material & $\mathrm{T}\left({ }^{\circ} \mathrm{C}\right)$ & $E(\mathrm{GPa})$ & $\nu(/)$ & $\sigma_{y}(\mathrm{MPa})$ \\
\hline Embedded PFC pipe & $\mathrm{CuCrZr}^{a}$ & 500 & 106 & 0.33 & 121 \\
& $\mathrm{Cu}$ & 500 & 90 & 0.33 & 26 \\
& $\mathrm{~W}$ & 1200 & 356 & 0.29 & 346 \\
& $\mathrm{~W}^{-}$recrystallized $^{b}$ & 2000 & 285 & 0.29 & 70 \\
Free PFC pipe & $\mathrm{CuCrZr}^{a}$ & 200 & 123 & 0.33 & 155 \\
Non-PFC pipe & $\mathrm{AISI}^{a} 316 \mathrm{~L}$ & 200 & 185 & 0.30 & 137 \\
Inlet/outlet pipe & AISI-316L & 200 & 185 & 0.30 & 137 \\
Cassette channel & Eurofer97 & 200 & 207 & 0.30 & 497 \\
\hline
\end{tabular}

${ }^{a} \mathrm{CuCrZr}-\mathrm{IG}$ (here IG - ITER Grade) alloy with typical treatment associated with First Wall and Divertor manufacturing cycles [17].

${ }^{b}$ Ultimate tensile strength of recrystallized tungsten is used as yield stress assuming a pre-hardened state [18].

Isotropic ideal plastic behaviour (e.g., with zero strain hardening) is assumed conservatively for all considered materials. In addition to the magnetic force density $f>0$ applied volumetrically within the pipe wall in the downward direction, a constant pressure of $p=50$ bar is assumed inside all pipe models [10].

Figures 4 and 5 present two plane-strain FE models with the equivalent plastic strains and von Mises stresses calculated for, respectively, the nonPFC and inlet/outlet pipe segments. The results are shown for three representative magnetic force densities, denoted by $f_{>0}, f_{50 \%}$ and $f_{100 \%}$, which represent critical values that provide respectively the onset, $50 \%$ and $100 \%$ area coverage of the plasticity along the pipe cross-section that is in contact with the cassette. The values are listed in Tab. 3. Due to employed conservatism in the assumed ideal plastic behaviour, the $f_{50 \%}$ is proposed to be a reasonably good measure of the mechanical damage initiation in simplysupported pipes. A comparison between the two pipe models demonstrates 


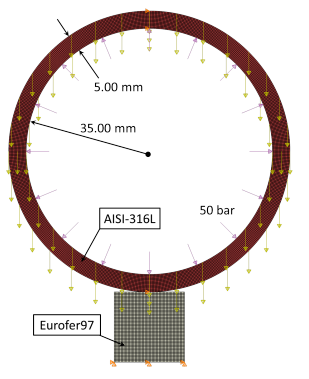

(a)

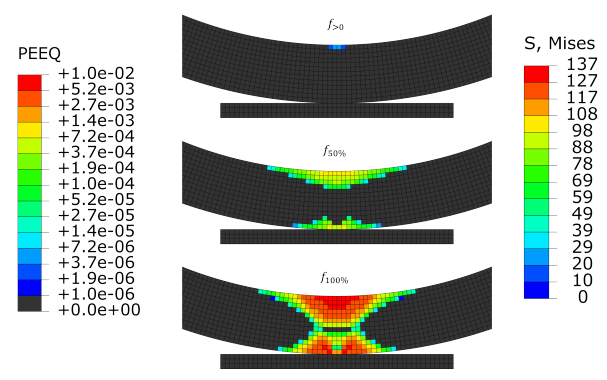

(b)

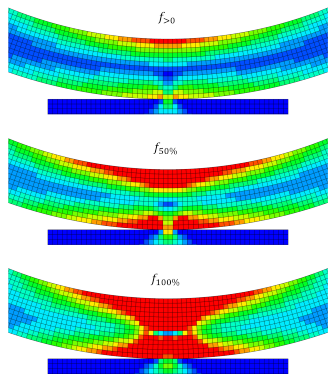

(c)

Figure 4: (a) FE model used to simulate a non-PFC pipe in contact with the cassette using a plane-strain approximation. Indicated are material sections, dimensions and two loadings (inner pipe pressure and downward body pipe force). Equivalent plastic strain PEEQ (b) and von Mises stress (c) are shown for three force densities $f_{>0}=0.06 \mathrm{~N} / \mathrm{mm}^{3}, f_{50 \%}=$ $0.09 \mathrm{~N} / \mathrm{mm}^{3}$ and $f_{100 \%}=0.13 \mathrm{~N} / \mathrm{mm}^{3}$ representing the critical values for respectively the onset, $50 \%$ and $100 \%$ area coverage of the plasticity along the lower pipe cross-section.

a well-known fact that smaller pipes (with same wall thickness) withstand larger forces (and therefore larger currents), which can be attributed to higher thickness-to-radius ratio.

Table 3: Calculated critical magnetic force densities $f_{>0}, f_{50 \%}$ and $f_{100 \%}$ corresponding to three different damage criteria: onset, $50 \%$ and $100 \%$ area coverage of the plasticity along the lower pipe cross-section. Note that embedded PFC pipe values have been reduced by five, which is an average number of monoblocks supported by a single support, see Fig. 6 .

\begin{tabular}{llll}
\hline Region & $f_{>0}\left(\mathrm{~N} / \mathrm{mm}^{3}\right)$ & $f_{50 \%}\left(\mathrm{~N} / \mathrm{mm}^{3}\right)$ & $f_{100 \%}\left(\mathrm{~N} / \mathrm{mm}^{3}\right)$ \\
\hline Embedded PFC pipe & 1.80 & 1.90 & 2.00 \\
Free PFC pipe & 0.80 & & \\
Non-PFC pipe & 0.06 & 0.09 & 0.13 \\
Inlet/outlet pipe & 0.05 & 0.07 & 0.11 \\
\hline
\end{tabular}

To perform an accurate integrity analysis also for the embedded PFC pipe, the realistic modeling of the tungsten monoblock around the PFC pipe is employed following Fig. 6. It is assumed, however, that the applied halo current is flowing only through the PFC pipe and not through the monoblocks $\left(I_{W}=0\right)$, which is believed to be a conservative approximation. The corresponding plane-strain FE model is shown in Fig. 7(a) with material parameters given in Tab. 2. 


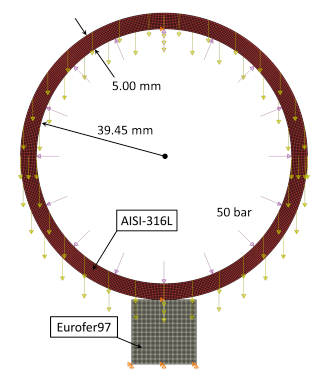

(a)

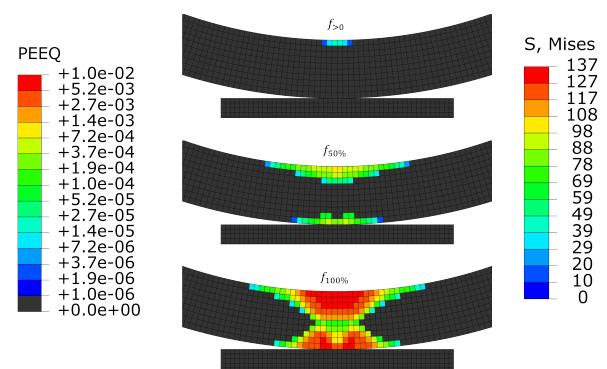

(b)

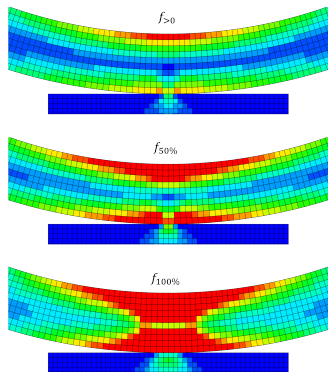

(c)

Figure 5: (a) FE model used to simulate an inlet/outlet pipe in contact with the cassette using a plane-strain approximation. Indicated are material sections, dimensions and two loadings (inner pipe pressure and downward body pipe force). Equivalent plastic strain PEEQ (b) and von Mises stress (c) are shown for three force densities $f_{>0}=0.05 \mathrm{~N} / \mathrm{mm}^{3}$, $f_{50 \%}=0.07 \mathrm{~N} / \mathrm{mm}^{3}$ and $f_{100 \%}=0.11 \mathrm{~N} / \mathrm{mm}^{3}$ representing the critical values for respectively the onset, $50 \%$ and $100 \%$ area coverage of the plasticity along the lower pipe cross-section.

In a similar manner as before, the integrity of the PFC segment is investigated by evaluating the amount of plasticity deployed in the model as a function of the applied downward magnetic body force within the pipe wall. The resulting equivalent plastic strains and von Mises stresses are shown in Fig. 7 for $f_{>0}=9 \mathrm{~N} / \mathrm{mm}^{3}$, which indicates the onset of plasticity in the copper interlayer. Note that the corresponding $f_{50 \%}$ and $f_{100 \%}$ values are similar in size to $f_{>0}$ as indicated in Tab. 3. Interestingly, the value of $f_{>0}=9 \mathrm{~N} / \mathrm{mm}^{3}$ for the PFC segment is two orders of magnitude higher than $f_{>0}=0.06 \mathrm{~N} / \mathrm{mm}^{3}$ for the simply-supported non-PFC pipe. This is purely due to perfect geometrical embedment of the PFC pipe into much stiffer and stronger tungsten monoblock.

The value of $9 \mathrm{~N} / \mathrm{mm}^{3}$, when multiplied by the volume of the PFC pipe segment $\left(\sim 900 \mathrm{~mm}^{3}\right)$, gives a total force of $8 \mathrm{kN}$, which is in good agreement with the requirement [19] that each monoblock support should be able to sustain $F_{c}=9 \mathrm{kN}$ of tensile force ${ }^{4}$. This amount of force can be induced, for example, when a total current of $I=F_{c} / l B \approx 21 \mathrm{kA}$ is flowing through a PFC pipe, where $l \approx 70 \mathrm{~mm}$ is the distance between the two PFC supports (see Fig. 6) and $B=6 \mathrm{~T}$ is the expected magnetic field at the outer target

\footnotetext{
${ }^{4}$ Note that the same critical force $f_{>0}=9 \mathrm{~N} / \mathrm{mm}^{3}$ is obtained here for the applied upward (tensile) force direction.
} 

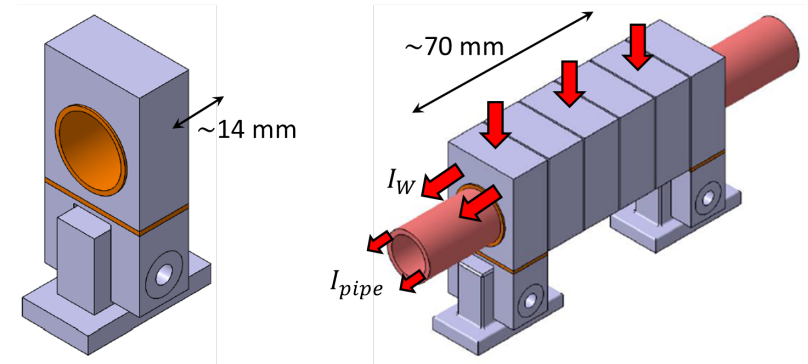

Figure 6: PFC segment with indicated dimensions and electrical current paths. In the mechanical integrity analysis, the current is conservatively assumed to flow only through the pipe section $\left(I_{W}=0\right)$.

position. Note that $\sim 21 \mathrm{kA}$ per $\mathrm{PFC}$ pipe is much larger than $\sim 2 \mathrm{kA}$ expected from the VDE-IV event (89 kA distributed evenly over 43 outer PFC pipes). This means that the PFC segment is well designed to withstand the most extreme electromagnetic forces.

The last mechanical analysis addresses the bending of a single monoblockfree PFC pipe segment located in the upper curved section of the outer target, where the highest electrical currents $(89 \mathrm{kA}$ in total) are anticipated during the VDE-IV slow down event. The pipe segment is analysed in Fig. 8 for inward force direction and clamped boundary conditions at both free ends. Damage initiation, this time due to pipe bending, is attributed again to the onset of plasticity at critical force density $f_{>0}=0.8 \mathrm{~N} / \mathrm{mm}^{3}$ (the correspond$\operatorname{ing} f_{50 \%}$ and $f_{100 \%}$ values are obsolete for this case). Note that the same critical force $f_{>0}=0.8 \mathrm{~N} / \mathrm{mm}^{3}$ is obtained for the applied outward force direction representing the opposite current or magnetic field direction. Although the obtained critical force is an order of magnitude smaller than the corresponding force in the embedded PFC pipe, it is nevertheless an order of magnitude larger than the critical forces calculated in the two simply-supported pipes. This suggests that PFC pipe bending will not be the limiting mechanical constraint in the current design. Note also that maximum pipe deflection at the critical force density is only $\sim 0.2 \mathrm{~mm}$.

Given the values for $f_{>0}, f_{50 \%}$ and $f_{100 \%}$ in Tab. 3, the corresponding critical current densities $j_{>0}, j_{50 \%}$ and $j_{100 \%}$ can be estimated from the relation $\mathbf{f}=\mathbf{j} \times \mathbf{B}$. Current densities are listed in Tab. 4 for the assumed magnetic field of $B=6 \mathrm{~T}$, which is roughly the expected field at the outer divertor po- 


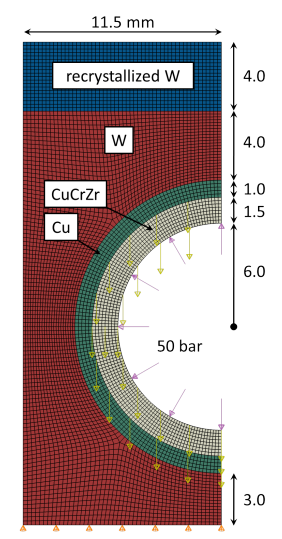

(a)

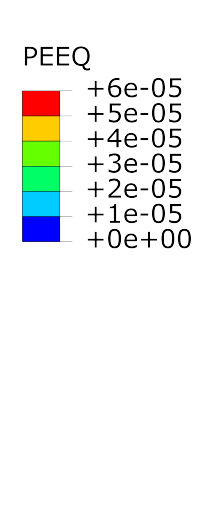

(b)

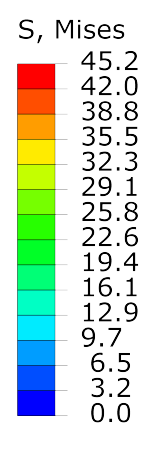

(c)

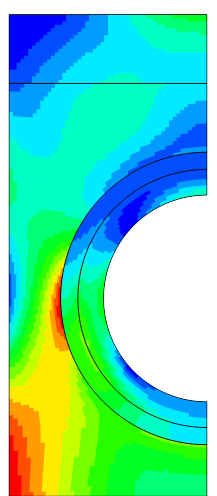

Figure 7: (a) FE model used to simulate the symmetric half of the PFC segment in a planestrain approximation. Indicated are material sections, dimensions and two loadings (inner pipe pressure and downward body pipe force). (b) Equivalent plastic strain PEEQ showing the initiation of plasticity in the copper interlayer at force density $f_{>0}=9 \mathrm{~N} / \mathrm{mm}^{3}$. (c) Von Mises stress field (in MPa) for the same $f_{>0}=9 \mathrm{~N} / \mathrm{mm}^{3}$.

sition where highest pipe currents are anticipated. Since the realistic toroidal magnetic fields vary along the divertor (from 5.5 T to 8.5 T), some margins need to be added to the values shown in Tab. 4.

Table 4: Critical current densities $j_{>0}, j_{50 \%}$ and $j_{100 \%}$ calculated from the corresponding critical magnetic force densities $f_{>0}, f_{50 \%}$ and $f_{100 \%}$ in Tab. 3 assuming external magnetic field of $B=6 \mathrm{~T}$, which is roughly the expected field at the outer divertor position where highest pipe currents are anticipated.

\begin{tabular}{llll}
\hline Region & $j_{>0}\left(\mathrm{~A} / \mathrm{mm}^{2}\right)$ & $j_{50 \%}\left(\mathrm{~A} / \mathrm{mm}^{2}\right)$ & $j_{100 \%}\left(\mathrm{~A} / \mathrm{mm}^{2}\right)$ \\
\hline Embedded PFC pipe & 300 & 317 & 333 \\
Free PFC pipe & 133 & & \\
Non-PFC pipe & 10 & 15 & 22 \\
Inlet/outlet pipe & 8 & 12 & 18 \\
\hline
\end{tabular}

\subsection{Thermal considerations}

In this section, the criterion for water boiling is discussed when accounting for Joule heating of the water-cooling pipes. Water boiling seems to be the most limiting scenario occurring at relatively low temperatures (water boils 


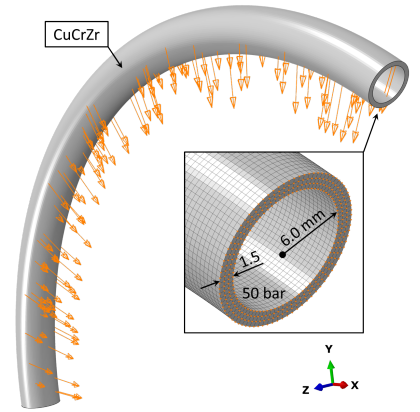

(a)

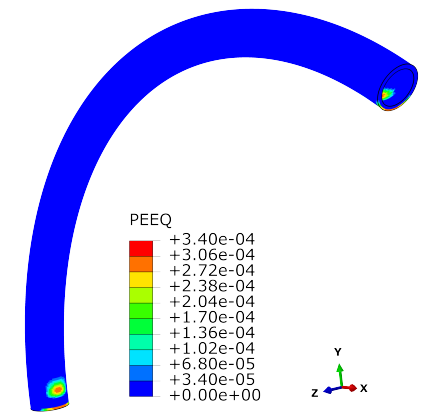

(b)

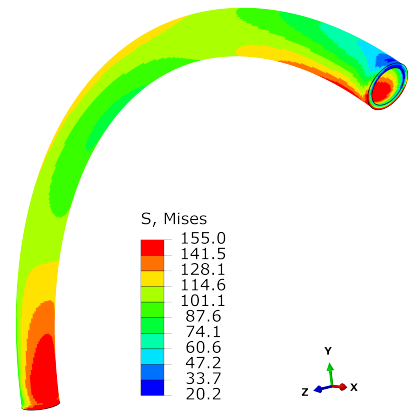

(c)

Figure 8: (a) 3D FE model of a monoblock-free PFC pipe located in the upper curved section of the outer target. Indicated are material section, pipe dimensions, clamped boundary conditions of free ends and two loadings (inner pipe pressure and inward body pipe force). (b) Equivalent plastic strain PEEQ showing the initiation of plasticity at force density $f_{>0}=0.8 \mathrm{~N} / \mathrm{mm}^{3}$. (c) Von Mises stress field (in MPa) for the same $f_{>0}=$ $0.8 \mathrm{~N} / \mathrm{mm}^{3}$.

at $264^{\circ} \mathrm{C}$ at 50 bar pressure [20]). Since electrical currents distribute more or less evenly across the pipe cross-section, thus heating the pipe uniformly, small thermal stresses are expected in the long straight pipe parts. However, sharp corners and other geometrical discontinuities with both large current densities and large density gradients can suffer from thermal stresses or even local material melting. These are not studied here.

When addressing the water-boiling conditions, the adiabatic limit is conservatively assumed where no heat is allowed to exchange between the pipe wall and the water. It is also assumed that water boiling initiates when the pipe wall is heated to $264^{\circ} \mathrm{C}$. From the energy conservation principle, the following formula for critical current density is derived

$$
j_{c}=\sqrt{\frac{\rho c_{p} \Delta T}{\rho_{e l} t_{C Q}}}
$$

where $\rho, c_{p}, \Delta T, \rho_{e l}$ and $t_{C Q}$ stand for the mass density, specific heat, temperature increase, electrical resistivity and current quench time, respectively. Material parameters are listed in Tab. 5 for the two considered pipe materials.

The resulting critical current densities are shown in Tab. 6 for the three different current quench time lengths $t_{C Q}$. Note that $t_{C Q}=200 \mathrm{~ms}$ was used in the calculation of VDE-IV slow down halo currents [8]. This value is also considered hereafter. 
Table 5: Material properties (mass density $\rho$, specific heat $c_{p}$ and electrical resistivity $\rho_{e l}$ ) of $\mathrm{CuCrZr}$ and AISI-316L materials obtained at two temperatures $T=140^{\circ} \mathrm{C}$ and $264^{\circ} \mathrm{C}$ from $[14,15,17]$, representing the coolant temperature at operating conditions [10] and water boiling temperature at 50 bar [20], respectively.

\begin{tabular}{lllll}
\hline Material & $T\left({ }^{\circ} \mathrm{C}\right)$ & $\rho\left(\mathrm{kg} / \mathrm{m}^{3}\right)$ & $c_{p}(\mathrm{~J} / \mathrm{KgK})$ & $\rho_{e l}(\mu \Omega \mathrm{m})$ \\
\hline $\mathrm{CuCrZr}$ & 140 & 8845 & 401 & 0.052 \\
& 264 & 8784 & 413 & 0.059 \\
AISI-316L & 140 & 7883 & 510 & 0.86 \\
& 264 & 7831 & 532 & 0.94 \\
\hline
\end{tabular}

Table 6: Critical current densities $j_{c}$ calculated from Eq. (1) and Tab. 5 for different current quench time lengths $t_{C Q}$.

\begin{tabular}{lll}
\hline Material & $t_{C Q}(\mathrm{~ms})$ & $j_{c}\left(\mathrm{~A} / \mathrm{mm}^{2}\right)$ \\
\hline CuCrZr & 50 & 385 \\
& 200 & 192 \\
& 1000 & 86 \\
AISI-316L & 50 & 103 \\
& 200 & 51 \\
& 1000 & 23 \\
\hline
\end{tabular}

In comparison to AISI-316L steel, approximately four times larger current densities are needed in $\mathrm{CuCrZr}$ alloy to produce the same amount of Joule heat. This factor originates primarily from the large differences in electrical resistivity of both materials.

\subsection{Summary of critical current densities}

The results of the above mechanical and thermal analyses are summarized in Tab. 7 where the lower of the two critical current densities is retained for each pipe region. From this, the following preliminary conclusions can be drawn:

- Admissible electrical currents through the water-cooling pipes will be limited by the structural integrity of the simply-supported pipes when uniformly pressed against the cassette body. 
Table 7: Summary of critical current densities and corresponding limiting criteria on different pipe regions.

\begin{tabular}{lll}
\hline Region & $j_{c}\left(\mathrm{~A} / \mathrm{mm}^{2}\right)$ & Criterion \\
\hline Embedded PFC pipe & 192 & Water boiling \\
Free PFC pipe & 133 & Pipe bending \\
Non-PFC pipe & 15 & Pipe compression \\
Inlet/outlet pipe & 12 & Pipe compression \\
\hline
\end{tabular}

- The structural integrity of these pipes can be significantly improved by optimizing their support structures, just like in embedded PFC pipes.

- Joule overheating of the pipes is less likely to occur also due to relatively large temperature difference between the operational and water boiling conditions.

In the following section, the identified critical current densities from Tab. 7 will be used to determine the optimal shunt resistances $\left(R_{s}\right)$ between the divertor cassette and water-cooling pipes.

\section{CURRENT AND VOLTAGE DISTRIBUTIONS}

In this section, the results of 3D electrical steady-state analyses are presented and discussed in the context of the previously identified critical pipe current densities (Tab. 7).

Figure 9 presents the calculated current pipe densities when the cooling pipes are electrically isolated from the cassette $\left(R_{s} \rightarrow \infty\right)$. It can be observed that almost half of the non-PFC pipes are overloaded; four regions are found in which the current density is exceeding the critical values as indicated with arrows in Fig. 9.

Interestingly, the currents in the $\mathrm{PFC}$ region remain well below the limiting values of 192 and $133 \mathrm{~A} / \mathrm{mm}^{2}$. This confirms the preliminary conclusion given in Sec. 3.3 that simply-supported pipes will be the weakest link and will therefore dictate the safe shunt value $R_{s, \max }$.

\subsection{Maximum shunt value}

In the following, the calculated current density distributions are shown for the two setups of shunt values: $R_{s}=1 \mathrm{~m} \Omega$ (Fig. 10) and $600 \mu \Omega$ (Fig. 11). In 


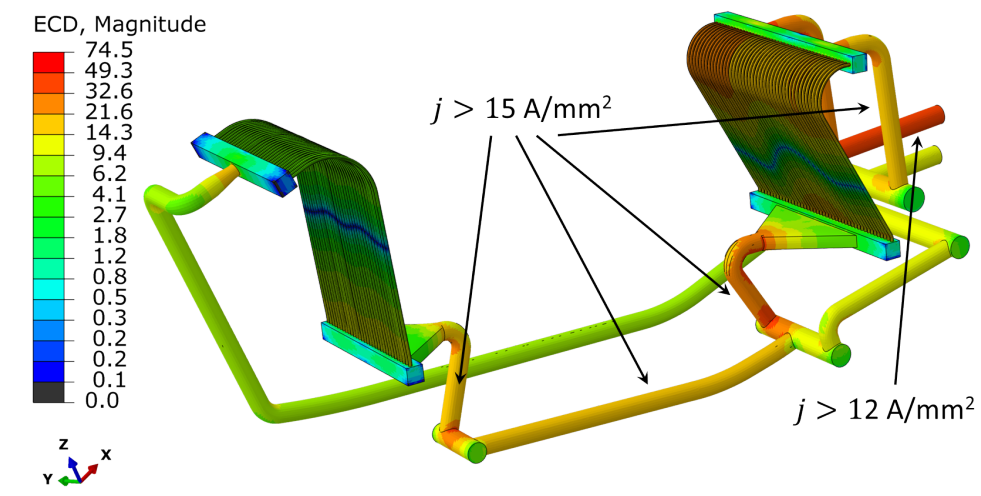

Figure 9: Results for the electrical current density (ECD in $\mathrm{A} / \mathrm{mm}^{2}$ ) for the applied $30 / 89 \mathrm{kA}$ current and assuming perfect isolation from the cassette $\left(R_{s} \rightarrow \infty\right)$. Logarithmic scale is used. Currents through non-PFC and inlet/outlet pipes exceed the critical values.

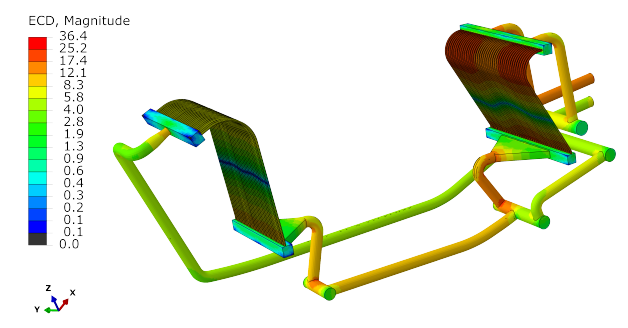

(a)

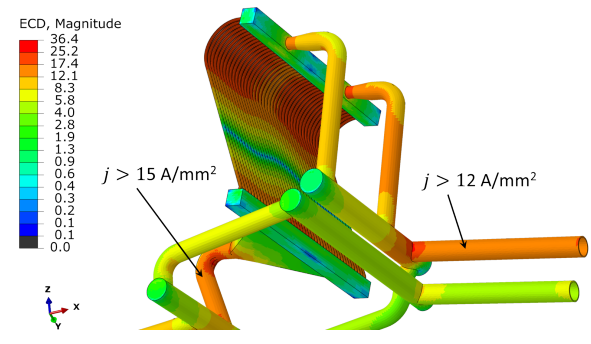

(b)

Figure 10: Results for the electrical current density (ECD in $\mathrm{A} / \mathrm{mm}^{2}$ ) for the applied $30 / 89 \mathrm{kA}$ current and using four shunt elements with $R_{s}=1 \mathrm{~m} \Omega$. Logarithmic scale is used. Indicated are pipe regions where the current slightly exceeds the critical value.

each setup, all four shunts are assumed to have the same resistivity, $R_{s, I T}=$ $R_{s, I B}=R_{s, O T}=R_{s, O B}=R_{s}$ (see Fig. 1 for the locations of the shunts). Using the same shunt values within each target $\left(R_{s, I T}=R_{s, I B}\right.$ and $R_{s, O T}=$ $\left.R_{s, O B}\right)$ implies that current load is more evenly distributed along the target PFC pipes.

It can be observed from Fig. 10 that $1 \mathrm{~m} \Omega$ shunts deliver slightly too large currents through the outlet pipe and short vertical non-PFC pipe; the other regions, however, seem to be safely below the limiting values. Note that $1 \mathrm{~m} \Omega$ shunts could become acceptable if either electrical grounding, pipe thickness or its supporting structure is optimized. 


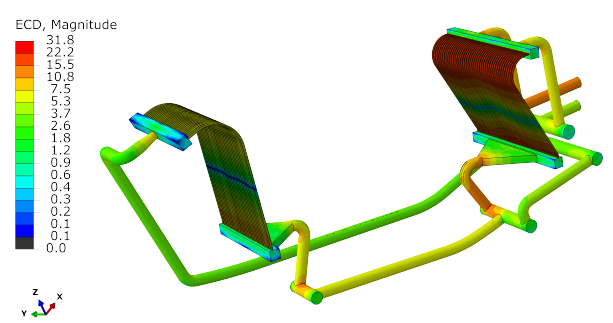

(a)

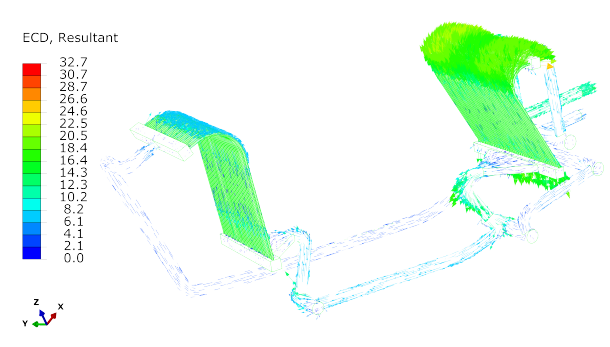

(c)
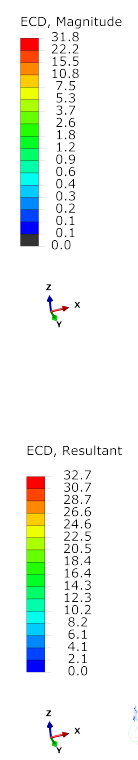

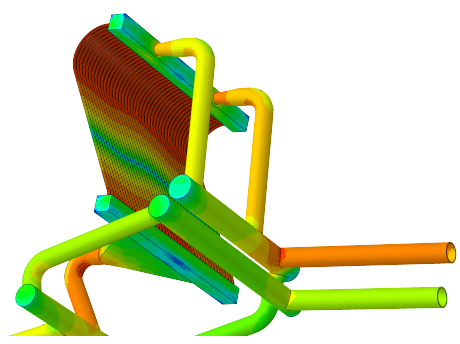

(b)

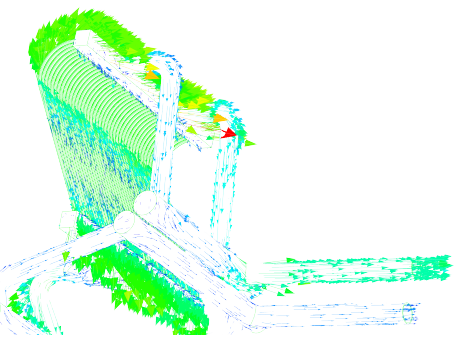

(d)

Figure 11: Results for the electrical current density (ECD in $\mathrm{A} / \mathrm{mm}^{2}$; both magnitude and vector fields are shown) for the applied $30 / 89 \mathrm{kA}$ current and using four shunt elements with $R_{s}=600 \mu \Omega$. Logarithmic scale is used in the top row. Practically all currents are below the critical values.

By iteration, the optimal shunt value is finally identified as $R_{s} \sim 600 \mu \Omega$, see Fig. 11. In this case, the current through the outlet pipe is just below the critical value of $12 \mathrm{~A} / \mathrm{mm}^{2}$, except on very small area at a pipe intersection where local currents can rise up to $\sim 20 \mathrm{~A} / \mathrm{mm}^{2}$ due to geometrical discontinuities. However, these regions are believed to be too small to considerably affect the integrity of the pipe.

Note that the largest current densities $\left(j>15 \mathrm{~A} / \mathrm{mm}^{2}\right)$ are observed on the outer PFC pipes, in particular, in the upper curved monoblock-free region. However, the bending of these pipes in external magnetic field is not critical since predicted current densities are well below the critical value $\left(133 \mathrm{~A} / \mathrm{mm}^{2}\right)$. As mentioned earlier, higher current concentrations are also observed locally at pipe intersections due to geometrical discontinuities.

\subsection{Estimated thermo-current shunt voltage}

Finally, in Fig. 12 the potential distribution along the divertor cassette and cooling pipes is shown for the applied $150 \mathrm{~A}$ thermo-current when using 

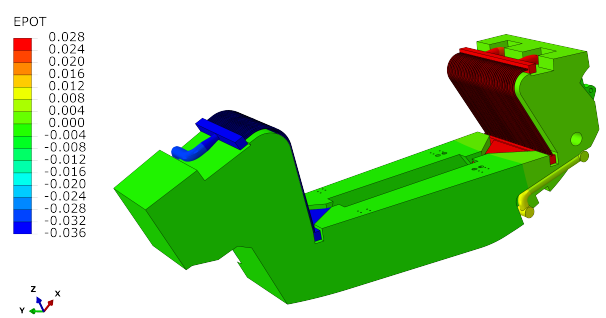

(a)

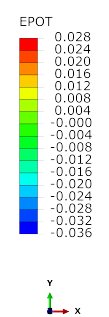

$\sqrt{x}$

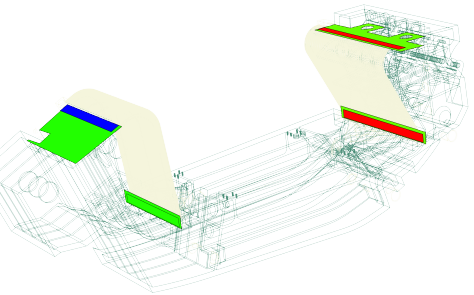

(b)

Figure 12: Results for the electrical potential (EPOT in V) for the applied $150 \mathrm{~A}$ current and using four shunt elements with $R_{s}=600 \mu \Omega$. A maximum voltage difference of $64 \mathrm{mV}$ is predicted between the inner and outer target plates.

the four shunt elements with maximum resistance of $R_{s}=600 \mu \Omega$ as identified above. The resulting shunt voltages on the inner and outer shunts are respectively $U_{s, \text { in }} \approx-36 \mathrm{mV}$ and $U_{s, \text { out }} \approx 28 \mathrm{mV}$, which give a $\sim 64 \mathrm{mV}$ voltage difference between the two target plates.

Although a $\sim 64 \mathrm{mV}$ potential difference should be detectable for plasma detachment control, even larger signals may be obtained by further optimization of the shunt values. For example, asymmetric distribution of shunt values could be used on inner and outer targets $\left(R_{s, I T}=R_{s, I B} \neq R_{s, O T}=\right.$ $\left.R_{s, O B}\right)$. However, the biggest gain in signal strength would be obtained by optimizing the divertor design. A few simple modifications could be already suggested based on the known limiting factors:

- The structural integrity of simply-supported pipes can be significantly improved by optimizing their support structures or even by increasing the pipe wall thickness.

- Maximum current densities along the inlet/outlet pipe sections can be further reduced by optimizing the electrical grounding.

\section{CONCLUSIONS}

Mechanical, thermal and electrical analyses have been performed in order to identify the number of shunts, their maximum resistances and their optimal locations in the isolated-target design model of the DEMO divertor. The analyses have adopted the recent DEMO baseline 2017 divertor model which by design precludes large pipe bending. 
The results of the analyses have shown that four shunt elements, located on four PFC junction pipes and with resistance of $\sim 600 \mu \Omega$ would protect the water-cooling pipes from the damaging effects of $30 / 89 \mathrm{kA}$ halo currents flowing through the inner/outer divertor targets during the extreme VDE-IV slow down disruption.

In the case of thermo-current measurement in DEMO, with much smaller target currents of $150 / 150 \mathrm{~A}$, the proposed shunts of $\sim 600 \mu \Omega$ would provide maximum voltage difference of $\sim 64 \mathrm{mV}$ between the inner and outer target plates. This signal amplitude seems to be large enough for reliable plasma attachment detection.

Further enhancements of the thermo-current signal may be achieved by assuming asymmetric distribution of shunt values on inner and outer PFC targets, or by considering small pipe design changes such as the implementation of specially designed pipe supports or the optimization of the electrical grounding specifically on the inlet/outlet pipes.

\section{ACKNOWLEDGMENTS}

This work has been carried out within the framework of the EUROfusion Consortium and has received funding from the Euratom research and training programme 2014-2018 and 2019-2020 under Grant Agreement No 633053 and cofunded by Slovenian Research Agency, grant P2-0405. The views and opinions expressed herein do not necessarily reflect those of the European Commission.

\section{References}

[1] A. Kallenbach, M. Bernert, T. Eich, J. Fuchs, L. Giannone, A. Herrmann, J. Schweinzer, W. Treutterer, Optimized tokamak power exhaust with double radiative feedback in asdex upgrade, Nuclear Fusion 52 (12). doi:10.1088/0029-5515/52/12/122003.

[2] G. Pautasso, L. Giannone, O. Gruber, A. Herrmann, M. Maraschek, K. Schuhbeck, The halo current in asdex upgrade, Nuclear Fusion 51 (4). doi:10.1088/0029-5515/51/4/043010.

[3] A. Kallenbach, A. Carlson, G. Pautasso, A. Peeters, U. Seidel, H.-P. Zehrfeld, Electric currents in the scrape-off layer in asdex upgrade, Journal of Nuclear Materials 290-293 (2001) 639-643. doi:10.1016/S00223115(00)00445-1. 
[4] G. Staebler, F. Hinton, Currents in the scrape-off layer of diverted tokamaks, Nuclear Fusion 29 (10) (1989) 1820-1824. doi:10.1088/0029$5515 / 29 / 10 / 017$.

[5] A. Chankin, S. Clement, L. de Kock, S. Erents, P. Harbour, J. Tagle, Parallel currents in the scrape-off layer of jet diverted discharges, Journal of Nuclear Materials 196-198 (C) (1992) 739-744. doi:10.1016/S00223115(06)80134-0.

[6] S. El Shawish, L. Giannone, A. Kallenbach, Study of electromagnetic disruption forces for plasma detachment measurements in demo, Fusion Engineering and Design 138 (2019) 372-378. doi:10.1016/j.fusengdes.2018.12.023.

[7] L. Giannone, S. El Shawish, A. Herrmann, A. Kallenbach, G. Mazzone, K. Schuhbeck, J. You, I. Zammuto, Conceptual study for detachment measurements in demo, Final report, EUROfusion, eFDA_D_2N6GTP (2017).

[8] C. Bachmann, Demo plant structural load specification, Final report, EUROfusion, eFDA_D_2MY7H3 (2019).

[9] V. Cocilovo, G. Ramogida, Simulation of electromagnetic vde plasma effects on divertor structures of demo, Fusion Engineering and Design 136 (2018) 1452-1460. doi:10.1016/j.fusengdes.2018.05.034.

[10] P. Di Maio, R. Burlon, M. Giardina, G. Mazzone, P. Chiovaro, A. Quartararo, E. Tomarchio, E. Vallone, Thermo-hydraulic assessment 2019, Final report, EUROfusion, eFDA_D_2MVK46 (2019).

[11] DEMO_DIVERTOR_2019_V1.1_2NCUJX_v1_0.stp, ｅFDA_D_2N24SN (2019).

[12] Simulia, ABAQUS 6.14-2 (2016).

[13] K. Mergia, N. Boukos, Structural, thermal, electrical and magnetic properties of eurofer 97 steel, Journal of Nuclear Materials 373 (1-3) (2008) 1-8. doi:10.1016/j.jnucmat.2007.03.267.

[14] I. Batra, G. Dey, U. Kulkarni, S. Banerjee, Microstructure and properties of a cu-cr-zr alloy, Journal of Nuclear Materials 299 (2) (2001) 91-100. doi:10.1016/S0022-3115(01)00691-2. 
[15] T. Chu, C. Ho, Thermal Conductivity and Electrical Resistivity of Eight Selected AISI Stainless Steels, Springer, Boston, MA, 1978.

[16] Https://www.lenntech.com/applications/ultrapure/conductivity/waterconductivity.htm.

[17] Iter structural design criteria for in-vessel components (sdc-ic), appendix a: Materials design limit data, Tech. rep., ITER, iTER_D_222RLN (2013).

[18] M. Li, J.-H. You, Structural impact of creep in tungsten monoblock divertor target at $20 \mathrm{mw} / \mathrm{m} 2$, Nuclear Materials and Energy 14 (2018) 1-7. doi:10.1016/j.nme.2017.12.001.

[19] V. Imbriani, U. Bonavolont, G. Di Gironimo, S. El Shawish, M. Fursdon, L. Giannone, D. Marzullo, G. Mazzone, E. Visca, J.-H. You, Insulated fixation system of plasma facing components to the divertor cassette in eurofusion-demo, Fusion Engineering and Design 158. doi:10.1016/j.fusengdes.2020.111710.

[20] Https://www.engineeringtoolbox.com/boiling-point-water-d_926.html. 